actually continually change their antigens. However, experiments by van Miervenne et al. (Annl Soc. belge. Med. trop 55, 1, 55, 25; 1976), using a fluorescent antibody technique to identify particular variants, have shown that clones rapidly become antigenically heterogeneous and that in every population there are a few individuals that do not bear the antigen characteristic of the population. These individuals are at an advantage when the immune response to the predominant antigen begins to become effective but are in their turn destroyed as they become dominant and have to give way to yet other variants.

Cross and his coworkers suggest that trypanosomes express a gene for and synthesise a single variant antigen at a time. Over evolutionary time a number of genes each coding for a particular variant have emerged by mutation, been selected for and have been accumulated giving the trypanosomes of the present time a vast repertoire of variants which they need to survive in their mammalian hosts. None of the available evidence contradicts this hypothesis and the next questions must be how the antigen switch is brought about and, when this is known, how it can be stopped. To those living and trying to raise cattle in the tsetse belt of Africa this is not just an academic question.

\section{Prenatal detection of thalassaemia}

from Sue Malcolm

THE various inherited haemoglobin disorders which collectively go under the name of thalassaemia are the most serious genetic diseases in many parts of the world. So the successful prenatal diagnosis of both the $\alpha$ and $\beta$ thalassaemias marks a significant advance in their prevention.

$\alpha$ thalassaemia (in which no $\alpha$ globin chains are produced) is now known to be caused by the deletion of the DNA coding for the $\alpha$ chain (Ottolenghi et al. Nature 251, 389; 1974; Taylor et al. Nature 251, 392; 1974), and Y. W. Kan and colleagues in San Francisco have now devised a method of determining whether the $\alpha$ globin genes are present in foetal DNA ( $\mathrm{New}$ Engl. J. Med. 295, 1165; 1976).

The foetal DNA was extracted from cultured amniotic fluid cells obtained by amniocentesis during the fifteenth week of pregnancy and cultured for a further five weeks to provide enough DNA for analysis. Radioactivelylabelled $\alpha$ globin complementary DNA synthesised on $\alpha$ globin mRNA was then hybridised to the foetal DNA. The results from this technique are quite clear cut but require the greatest precision in mixing the two DNAs and care in making the measurements.

At the ratios of cDNA to DNA used there should be three $\alpha$ globin minus strands (two from the cold foetal DNA and one from the cDNA) to every two plus strands in normal DNA, and therefore the radioactive probe should be $66 \%$ hybridised. If two $\alpha$ globin genes are deleted ( $\alpha$ thal I) the ratios would be two minus strands to one plus strand, and only $50 \%$ hybridisation found, and if three genes are deleted $(\mathrm{Hb} \mathrm{H}$ disease) the minus: plus ratio would be $3: 1$ and $33 \%$ hybridisation found. If in fact all four $\alpha$ genes are deleted in the foetus (hydrops fetalis) no hybridisation would be expected. Unfortunately the $\alpha$ globin cDNA was only $80 \%$ pure, the remaining sequences being $\beta$ cDNA so even in a case of hydrops fetalis around $20 \%$ hybridisation would be found. A successful diagnosis therefore rests on the ability to distinguish between $20 \%$ and around $45 \%$ hybridisa tion $(\sim 125$ c.p.m.) since only the difference between hydrops fetalis and $\mathrm{HbH}$ is clinically important. In the case reported Kan successfully predicted that the foetus examined had $\alpha$ thalassaemia I. Later, twins were diagnosed and the parents had no hesitation in continuing the pregnancy since at least one foetus was known to be unaffected. In the event healthy twins, one male and one female, were born. At least some of the uncertainty in this type of diagnosis will be removed when cloned, and therefore pure, human globin sequences become available.

An even more serious health problem is posed by the diseases associated with faulty $\beta$ globin chain production, $\beta$ thalassaemia and sickle-cell anaemia. Reports from a combined Boston and London study (Alter et al. New Engl. J. Med. 295, 1437; 1976) and from Kan's San Francisco group (Lancet i, 269; 1977) announce encouraging progress in the prenatal diagnosis of both these diseases. In both cases there is an error of $\beta$ globin expression rather than a gene deletion; in $\beta$ thalassaemia no $\beta$ globin chain is produced and in sickle-cell disease a mutant globin chain $\left(\beta_{\mathrm{s}}\right)$. Therefore analysis can only be carried out on cells which actually produce globin - the foetal blood cells.

The biochemical problem has been elegantly solved. The blood cells are incubated with ${ }^{3} \mathrm{H}$-leucine and the resultant globin chains analysed on carboxymethylcellulose chromatography columns. In the case of $\beta$ thalassaemia the $\beta / \gamma$ globin ratio is measured and in the case of sickle-cell disease the presence of $\beta_{\Lambda}$ or $\beta_{\mathrm{S}}$ is determined. Prenatal diagnosis is possible because even in the first trimester of pregnancy $10 \%$ of the $\beta$-like chains produced are $\beta$ rather than the foetal $\gamma$ globin.

Serious problems in interpretation could arise if the blood sample were contaminated with maternal cells. The Boston-London group overcame this problem by transfusing the mother to reduce her reticulocyte (globin-producing cell) count before foetal blood sampling and the San Francisco group used anti-i serum, an antibody that agglutinates foetal cells. Both groups detected homozygous $\beta$ thalassaemia and homozygous sickle-cell disease in utero and their results were confirmed after abortion.

However, both groups also found foetal blood sampling a hazardous procedure and altogether 6 out of 55 foetuses were lost as a result of attempting to sample foetal blood (three from each centre). The Boston-London group used placental aspiration when there was an anterior placenta and foetoscopy for a posterior placenta. The San Francisco workers used placental aspiration in both cases. The BostonLondon workers found foetoscopy less hazardous and since their very first attempts they have had far greater success. The one case in which they wrongly predicted a healthy baby was the result of an insufficient foetal blood sample.

Although the results of the two groups combined are very encouraging it is clear that attempts to take foetal blood during early stages of pregnancy can be very dangerous and the continuing use of these methods for prenatal diagnosis depends on this procedure's becoming much safer. As pointed out in an editorial in the British Medical Journal (531, 532; 1977) it will also be necessary to simplify the biochemical procedures and the apparatus involved before these techniques can be applied in the parts of the world where these diseases are common.

\section{Polarity time scale extended by 2 Myr}

from Peter J. Smith

Writ the possible exception of any very short events remaining undetected, the details of the geomagnetic polarity time scale based on radiometrically-dated subaerial igneous rocks of the past $4.5 \mathrm{Myr}$ have been known for some time. It would now clearly be desirable to extend this well-established 